\title{
Effects of Mechanical Vibration on Macrostructure and Mechanical Properties of AC4C Aluminum Alloy Castings
}

\author{
Naoki Omura ${ }^{1}$, Yuichiro Murakami ${ }^{1}$, Mingjun $\mathrm{Li}^{1}$, Takuya Tamura ${ }^{1}, \mathrm{Kenji} \mathrm{Miwa}^{1}$, \\ Hideki Furukawa ${ }^{2}$, Masayuki Harada ${ }^{2}$ and Mitsuyoshi Yokoi ${ }^{2}$ \\ ${ }^{1}$ National Institute of Advanced Industrial Science and Technology, Nagoya 463-8560, Japan \\ ${ }^{2}$ Kotobuki Kinzoku Kogyo Co. LTD., Seki 501-3928, Japan
}

The gravity die casting of the AC4C aluminum alloy was conducted when mechanical vibration was incorporated. The specimen with a specification of $25 \mathrm{~mm}$ in diameter and $210 \mathrm{~mm}$ in length were solidified at various vibration frequencies so as to examine the effect of the vibration on the grain size, the casting defect distribution, and mechanical properties. In comparison with the grains formed in the as-cast state without vibration, the grains in the inner area of a specimen became finer after mechanical vibration. The columnar structure remained in its outer region under all vibration frequency. The average density of specimen increased by imposition the mechanical vibration. The casting defects involved in the specimen reduced and became smaller with the increase of vibration frequency. The scattering in mechanical properties of specimens cast with mechanical vibration decreased because of the decrease in casting defects. [doi:10.2320/matertrans.F-M2009822]

(Received June 26, 2009; Accepted July 30, 2009; Published September 30, 2009)

Keywords: mechanical vibration, aluminum, alloy, casting defect, mechanical properties, refining, X-ray computed tomography

\section{Introduction}

Casting process of aluminum alloys is widely used in industrial engineering as the technique can fabricate the products with complex shape in lower superheat in a simple processing run. Because of the serious global warming in recent years, the material substitution from iron and steel to aluminum alloy is positively promoted due to the ecofriendly merit of the aluminum alloy. It is expected that the application of aluminum alloy castings continues to expand in the future.

Casting methods of aluminum alloys are broadly classified into three categories, i.e., gravity die casting, low-pressure die casting, and high-pressure die casting depending on the applied pressure to the melt. Because of the features such as short processing time, high productivity, and high accuracy, high-pressure die casting is most widely used in each field, e.g., the automobile industry, and the amount of production has been increased year by year. In terms of the gravity die casting and low-pressure die casting techniques, the total amount of product by these two methods increases every year though it is less than that of high-pressure die casting. ${ }^{1)}$

During gravity and low-pressure die casting processing, melt is poured very quietly and slowly into the cavity of a mold to avoid including gases. As a result, the products fabricated by these two methods have far fewer inner defects than those by the high-pressure die casting. This small amount of inner defects in the castings allows heat treatment and welding in the following processing. A striking disadvantage is that gravity die casting requires a large volume of riser to prevent the shrinkage defects, resulting in the long curing time and low material yield. Furthermore, this low cooling rate enables the grain coarsening and the solute element segregation. Hence, the grain refining agent, such as titanium, boron, carbon, and mother alloys including these elements, are widely used ${ }^{2-5)}$ in usual aluminum castings to refine and homogenize the macrostructure and the microstructure. But, these grain refining agents induce some problems not only in production but also in recycling. In production, both the grain refining agent and the addition process increase the cost of the final product. In recycling, on the other hand, the separation or removal of grain refining agents becomes difficult and it is a cost-added process. Because of the increasing concern on resource saving, energy saving, and recycling, a new process to refine and homogenize the cast structure is required. The increase in solidification rate and the imposition of vibration or agitation ${ }^{6-8)}$ are known as a method to refine and homogenize the castings structure.

The gravity and low-pressure die castings are mainly applied to large and thick products, so it is difficult to increase the solidification rate due to the thermal extraction constraints. Regarding vibration and agitation processes, ultrasonic vibration process, ${ }^{9,10)}$ electromagnetic stirring process, ${ }^{11,12)}$ electromagnetic vibration process, ${ }^{13,14)}$ and mechanical vibration process have been reported. In the ultrasonic vibration process, vibration area is very narrow, and it is necessary to insert and remove the ultrasonic horn in each casting. So the apparatus and casting process become more complex. In the electromagnetic stirring and the electromagnetic vibration, a huge equipment to generate the magnetic field is required, and the shape and size of products are limited due to the size of an induction coil and a magnet. In mechanical vibration process, on the other hand, a large or special device is not needed and the mechanical vibration can be readily imposed.

In this study, the columnar AC4C aluminum alloy rods were cast using the gravity die casting technique when mechanical vibration was imposed at the frequency range of $0 \sim 157 \mathrm{~Hz}$. The effects of mechanical vibration on grain size, distribution of casting defects, and mechanical properties were investigated. 


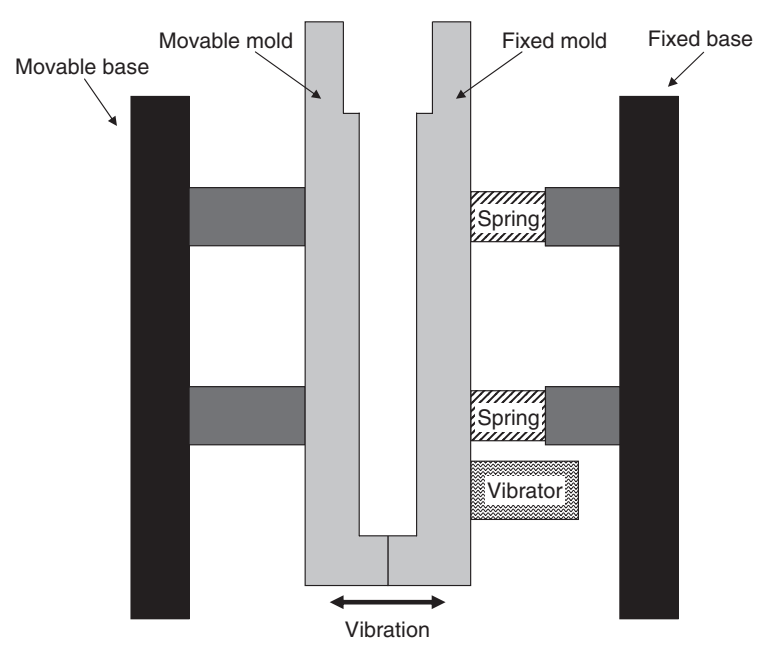

Fig. 1 Schematic diagram of the experimental apparatus. The bar with arrows at the extremes indicates the motion direction of the mold during vibration.

Table 1 Vibration performance of the vibrator for mechanical vibration casting.

\begin{tabular}{cccccc}
\hline $\begin{array}{c}\text { Air pressure } \\
(\mathrm{MPa})\end{array}$ & 0 & 0.2 & 0.3 & 0.4 & 0.47 \\
\hline $\begin{array}{c}\text { Centrifugal force } \\
(\mathrm{kN})\end{array}$ & 0 & 0.7 & 0.97 & 1.23 & 1.37 \\
\hline $\begin{array}{c}\text { Frequency } \\
(\mathrm{Hz})\end{array}$ & 0 & 112 & 132 & 148 & 157 \\
\hline
\end{tabular}

Table 2 Chemical composition of the AC4C aluminum Alloy.

\begin{tabular}{cccccccc}
\hline $\mathrm{Si}$ & $\mathrm{Mg}$ & $\mathrm{Ti}$ & $\mathrm{Fe}$ & $\mathrm{Ni}$ & $\mathrm{Cu}$ & $\mathrm{Zn}$ & $\mathrm{Al}$ \\
\hline 6.66 & 0.320 & 0.006 & 0.112 & 0.001 & 0.003 & 0.024 & Bal. \\
\hline
\end{tabular}

\section{Experimental Procedure}

\subsection{Experimental apparatus and vibration casting}

The experimental apparatus of the vibration casting is schematically shown in Fig. 1. The vibrator was directly placed at the bottom of the mold outer surface. The bar with arrows at the extremes indicates the motion direction of the mold during vibration. To prevent the opening of the mold by vibration, the fixed mold was held with spring. The vibrator (CH25A; EXEN, Tokyo, Japan) used in this study was oscillated using centrifugal force that was entailed in highspeed centrifugal motion of steel ball in circular space by air pressure. Thus, the vibration conditions such as frequency and centrifugal force could be easily controlled by changing the air pressure supplied. The vibration conditions used in this test are given in Table 1 .

The chemical composition of the AC4C aluminum alloy used in this experiment is shown in Table 2. Figure 2 depicts the shape of castings fabricated by mechanical vibration casting. Round bar samples $(25 \mathrm{~mm}$ in diameter and $210 \mathrm{~mm}$ in length) were cast using bottom gate plan. In this figure, the direction of the vibration is a vertical to the plane of this paper. Mechanical vibration casting was



(a) castings



(b) tensile specimen
Fig. 2 Schematic drawing of (a) castings and (b) a tensile test specimen.

carried out at $1033 \mathrm{~K}( \pm 15 \mathrm{~K})$ of the melt temperature and $573 \mathrm{~K}$ of the mold temperature. The imposition of mechanical vibration started before pouring, and stopped at $80 \mathrm{~s}$ after pouring.

\subsection{Density measurement and macrostructure observa- tion}

The casting specimens were cut into 7 successive parts along the longitudinal direction as shown in Fig. 2(a), and the density of each part was measured by the Archimedes method. To observe the macrostructure, each sample was cut parallel to the longitudinal direction, and ground following the standard procedure for metallographic preparation, and etched in a copper chloride solution.

\subsection{X-ray computer tomography (CT) analysis}

Inner defects of the as-cast specimens fabricated by mechanical vibration casting were observed using an X-ray CT system (SMX-225CT; Shimadzu, Kyoto, Japan). The conditions for the $\mathrm{CT}$ observation were as follows; the voltage and the current of the X-ray tube were $100 \mathrm{kV}$ and $30 \mathrm{~mA}$, respectively, the slice thickness was $0.1 \mathrm{~mm}$, and the cross-sectional resolution was $512 \times 512$ pixel. Figure 3 shows (a) a typical X-ray CT image and ((b) $\sim(d))$ the crosssectional photographs in the place corresponding to the $\mathrm{X}$-ray CT image. In the X-ray CT image, the area of high $\mathrm{X}$-ray absorption capacity is presented in white, while the area of low X-ray absorption capacity is expressed in black. In this study, therefore, the matrix (aluminum) area is indicated in white and internal defects such as pore are drawn in black. By comparing the CT image and the cross-sectional photographs, it can be readily seen that the independent defect was drawn clearly in black, and a area where some small defects concentrate was drawn in faint gray.

\subsection{Tensile test}

Mechanical properties of specimens cast with mechanical vibration were assessed by tensile test. As shown in Fig. 2(b), a tensile test specimen with a gauge section of $14 \mathrm{~mm}$ in diameter and $50 \mathrm{~mm}$ in length was machined. Tensile tests were carried out at room temperature at a constant crosshead speed of $3 \mathrm{~mm} / \mathrm{min}$. 

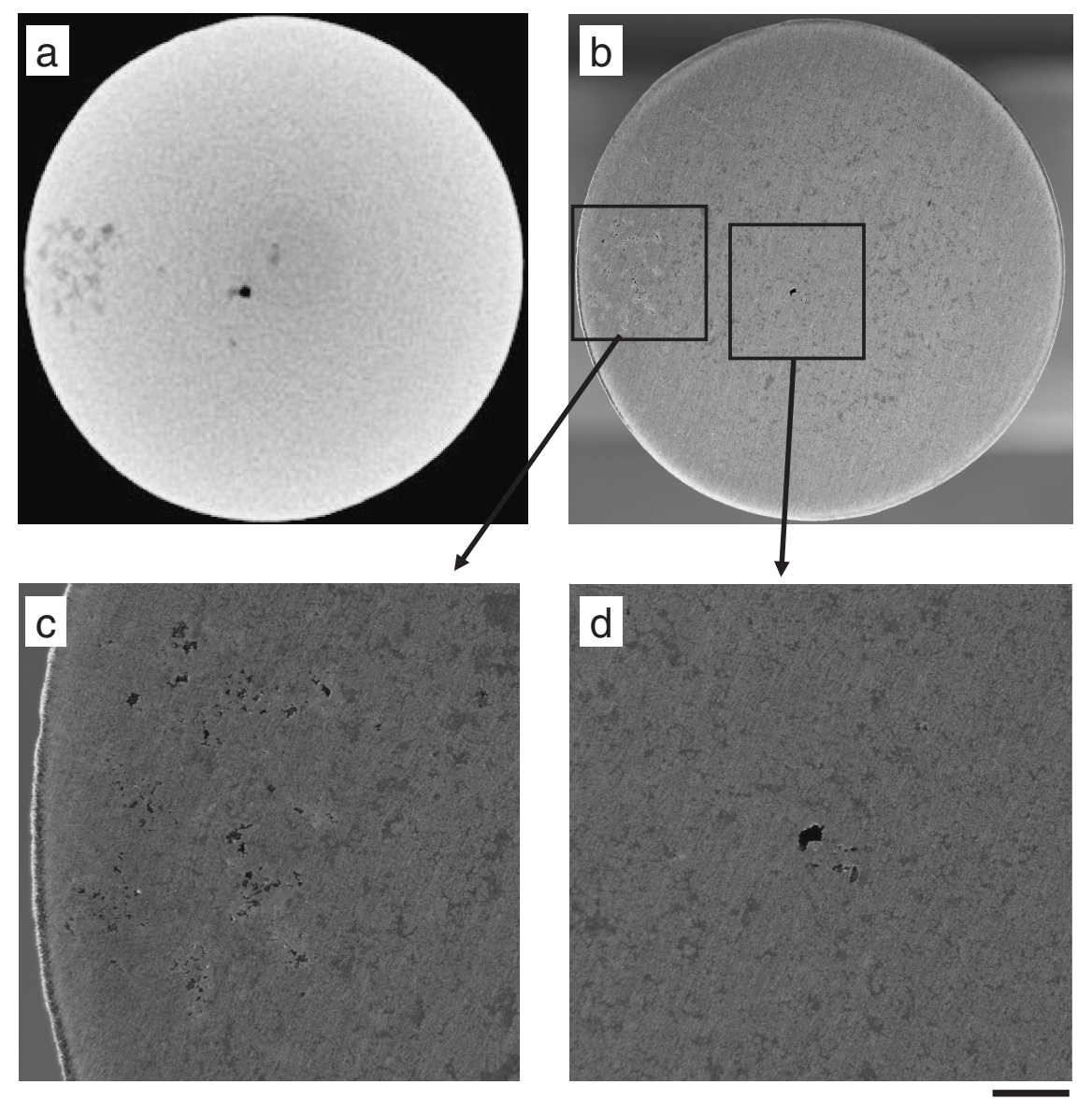

$1 \mathrm{~mm}$

Fig. 3 (a) A typical X-ray CT image of as-cast specimen and (b) the cross-sectional photograph corresponding to the area (a). (c) and (d) show the enlarged view of the area with defects.

\section{Results}

Figure 4 shows typical macrostructures of castings fabricated with or without mechanical vibration. In the specimen cast without mechanical vibration, as shown in Fig. 4(a), columnar structure and granular structure are observed in their outer and inner region respectively. Similarly, in the specimens cast with mechanical vibration, a mixed structure consisting of columnar in the outer region and granular in the inner region is observed. As seen above, there is no clear difference on the macrostructure by the addition of the mechanical vibration. On the other hand, the grain size is strongly affected by the mechanical vibration. By applying vibration, the grain size, especially the granular structure in the inner area becomes small. However, in this study, a clear grain size reduction by the increase of the vibration frequency is not observed.

Figure 5 presents the density of as-cast specimens at different vibration frequency. The calculated density of this material estimated by the chemical composition measured using spectroscopic analysis is $2679\left(\mathrm{~kg} / \mathrm{cm}^{3}\right)$ and is indicated by the solid line in the Fig. 5 . The density of the specimen cast without vibration varies with positions and indicates a remarkably low value at some positions. In the specimens cast with vibration, the position of very low density does not exist, though the density scattering in the position is observed. The average density slightly increases by mechanical vibration. There is no clear tendency of density scattering in every specimen. Furthermore, there is no strong relation between vibration frequency and the degree of the density scattering.

The X-ray CT images of specimens cast with and without mechanical vibration are shown in Fig. 6. The left part at each frequency shows the vertical section including the diameter, and right part shows the cross section at the middle of longitudinal of each seven part (shown in Fig. 2(a)). In the specimen without vibration, some faint gray areas and black areas are observed, indicating the existence of some concentrated small defects and independent defects. In the specimen mechanically vibrated at $112 \mathrm{~Hz}$, the gray area is not observed though some black areas are still maintained. At a higher frequency $(157 \mathrm{~Hz})$, both gray and black areas are not observed. This indicates that the casting defects are decreased and disappeared by the imposition of mechanical vibration. It is considered that as the fluidity of molten metal is increased by mechanical vibration, the supply of the molten metal to solidification shrinkage area is promoted, resulting in the casting defects are reduced and disappeared. In the case of gravity die casting, it is known that the effects of the riser decrease and the shrinkage cavities generate when 




(a) $0 \mathrm{~Hz}$

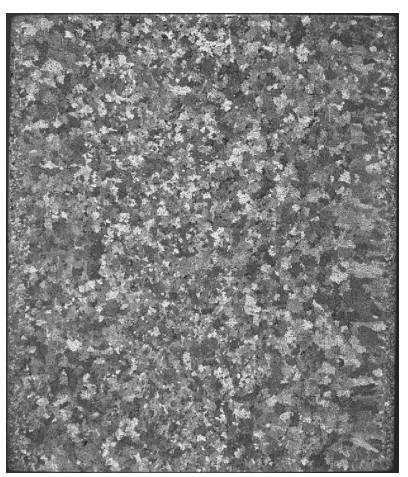

(b) $112 \mathrm{~Hz}$



(c) $132 \mathrm{~Hz}$

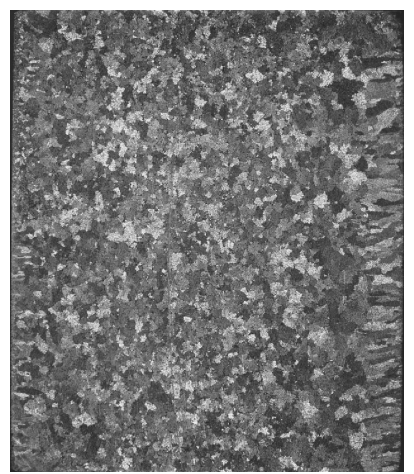

(d) $148 \mathrm{~Hz}$

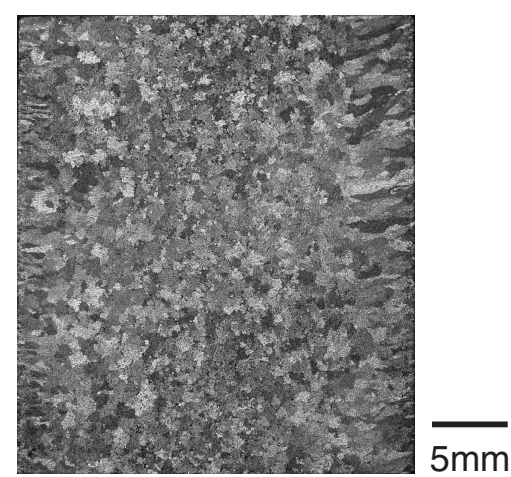

(e) $157 \mathrm{~Hz}$

Fig. 4 Macrostructures of specimens cast under different vibration frequencies of (a) $0 \mathrm{~Hz}$, (b) $112 \mathrm{~Hz}$, (c) $132 \mathrm{~Hz}$, (d) $148 \mathrm{~Hz}$ and (e) $157 \mathrm{~Hz}$

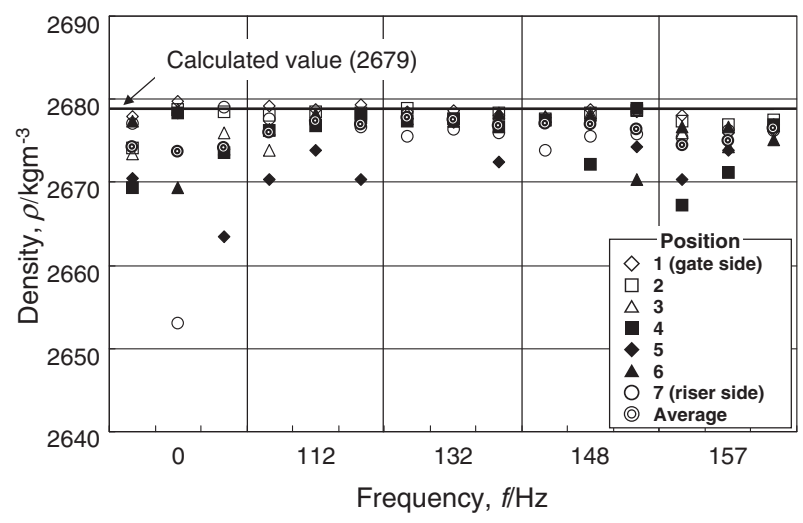

Fig. 5 Effect of vibration frequency on the density.

the solidified shells form and grow at the surface of riser and pouring cup. ${ }^{15)}$ In this process, it is also considered that the riser works effectively because the formation and growth of the solidified shell are inhibited by vibration.

Figure 7 represents the ultimate tensile strength (UTS) and the elongation of as-cast specimens. It should be noted that the UTS and the elongation of specimens at $0 \mathrm{~Hz}$ scattered widely. On the other hand, the UTS and the elongation of specimens cast with vibration hardly scatter. Furthermore, these properties slightly increase by the imposition of mechanical vibration. The UTS and the elongation of vibrated specimen are independent on the vibration frequency, and about $170 \mathrm{MPa}$ and $4.5 \%$, respectively.

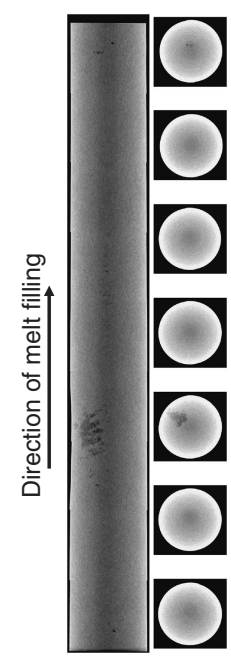

$\mathrm{OHz}$



$112 \mathrm{~Hz}$

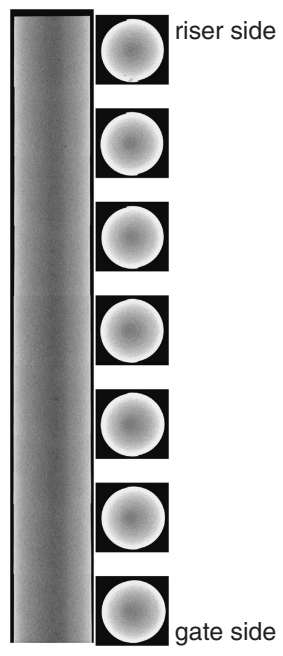

$157 \mathrm{~Hz}$
Fig. 6 Effect of vibration frequency on casting defects.

\section{Discussion}

The casting defects decreased with the increase of the vibration frequency, and were hardly observed in the specimen vibrated at $157 \mathrm{~Hz}$ (Fig. 6). However, the scattering in density and the average density of each sample was independent of vibration frequency, expect for the specimen without vibration. These results indicate that each vibrated specimen has similar volume of casting defects. In a CT 


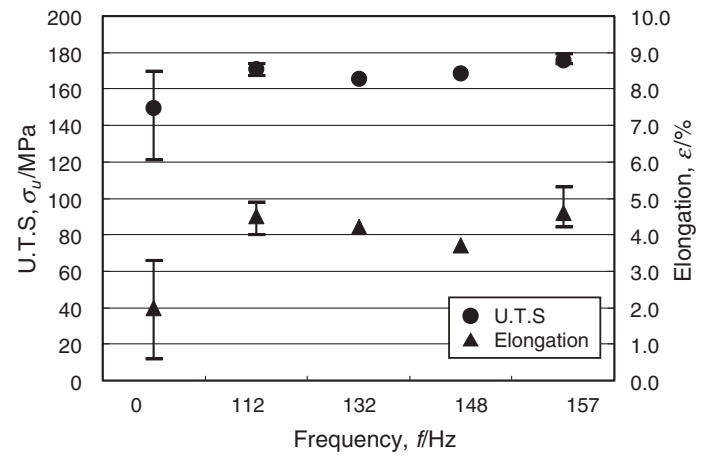

Fig. 7 Ultimate tensile strength ( $)$ and elongation $(\boldsymbol{\Delta})$ of casting specimens as a function of the vibration frequency.

image of this experiment, one pixel is represented by a square with the edge length of $0.1 \mathrm{~mm}$, so it is difficult to detect very small defects due to the limited resolution. The concentrated small defects, as shown in Fig. 3(c), can be expressed in the CT image in faint gray, but a very small independent defect can not be displayed easily. Thus, it is considered that very small defects exist and disperse in the specimen vibrated at $157 \mathrm{~Hz}$. So the average density of this specimen is identical to the specimens vibrated at a lower vibration frequency. These results indicate that mechanical vibration works to refine not only the macrostructure but also the casting defects of the products.

The specimen cast without vibration showed large scattering of the UTS and the elongation in tensile test (Fig. 7). Figure 8 presents (a) a fracture surface of specimen with the lowest UTS value in this study, and (b) an X-ray CT image corresponding to the fracture surface area. The circle drawn in dotted line in the CT image shows the size of tensile test specimen (X-ray CT analysis was carried out before machining). It is clearly seen that the fracture surface of this specimen contains the many small defects drawn in faint gray in the CT image. It is considered that this faint gray area is the origin of the fracture, and yields remarkable lower values of the UTS and the elongation. As shown in Fig. 6, such an area tended to exist in the specimen without vibration. This may be the reason that the UTS and the elongation of the as-cast specimens scattered greatly. In the specimen solidified with vibration, on the other hand, the scattering of mechanical properties was hardly observed. As mentioned above, many small defects might exist and disperse in these specimens though there were not observed in the CT images. Thus, it is considered that the very small defects have no influence on the mechanical properties.

By the imposition of mechanical vibration upon the gravity die casting, inner casting defects of products reduced and became very small and distributed uniformly throughout the entire volume of a specimen. Hence, the scattering of the mechanical properties such as UTS and elongation decreased remarkably. We believe that this process is very useful in the enhancement of the reliability of casting products.

\section{Conclusions}

Mechanical vibration was imposed on the gravity die casting of AC4C aluminum alloys. The effects on the macrostructure, the casting defect, and mechanical properties were investigated. The grain size in the inner area of columnar rod tends to decrease by the imposition of the mechanical vibration. Furthermore, the inner casting defects reduce and become smaller. As a result, the scattering of the mechanical properties of specimens cast with vibration decrease remarkably, and the elongation slightly increases.


$5 \mathrm{~mm}$

\section{(a) Fracture surface \\ (b) CT image at the fracture area}

Fig. 8 (a) Fracture surface of the specimen cast without vibration after tensile test. (b) X-ray CT image corresponding to fracture area (a). The dotted circle shows the size of specimen for tensile test. 


\section{REFERENCES}

1) H. Horikawa: J. Jpn. Inst. Light Met. 58 (2008) 3.

2) Y. C. Lee, A. K. Dahle, D. H. StJohn and J. E. C. Hutt: Mater. Sci. Eng. A 259 (1999) 43.

3) S. A. Kori, B. S. Murty and M. Chakraborty: Mater. Sci. Eng. A 283 (2000) 94.

4) T. Sritharan and H. Li: J. Mater. Proc. Tech. 63 (1997) 585.

5) C. T. Lee and S. W. Chen: Mater. Sci. Eng. A 325 (2002) 242.

6) Y. Kawaguchi, M. Sugamata and J. Kaneko: J. Jpn. Inst. Light Met. 47 (1997) 3 [in Japanese].
7) H. Fujii, M. Sugamata, J. Kaneko and M. Kubota: J. Jpn. Inst. Light Met. 50 (2000) 330 [in Japanese].

8) K. Miwa: J. Jpn. Inst. Light Met. 52 (2002) 611 [in Japanese].

9) Y. Osawa and A. Sato: J. JFS 72 (2000) 733 [in Japanese].

10) O. V. Abramov: Ultrasonics 25 (1987) 73.

11) J. Dong, J. Cui, X. Zeng and W. Ding: Mater. Lett. 59 (2005) 1502.

12) B. Zhang, J. Cui and G. Lu: Mater. Sci. Eng. A 355 (2003) 325.

13) Y. Mizutani, S. Kawai, K. Miwa, K. Yasue, T. Tamura and Y. Sakaguchi: Mater. Trans. 45 (2004) 1939-1943.

14) Y. Mizutani, Y. Ohura, K. Miwa, K. Yasue, T. Tamura and Y. Sakaguchi: Mater. Trans. 45 (2004) 1944-1948.

15) T. Takase: Foundry Engineering (AGNE) (1966) 138 [in Japanese]. 